\title{
POLÍTICAS Y CONCEPTOS DE CALIDAD: DILEMAS Y RETOS
}

\author{
José Dias SobrinHo*
}

* Es doctor en Ciencias Humanas. Fue vicerrector de postgrado de la Universidad Estadual de Campinas (Unicamp), presidió la Comisión Especial de Evaluación (2003, MEC, Brasil). Es profesor de Postgrado en Educación de la Universidad de Sorocaba y director de la Revista Avaliação. Autor de varios libros y artículos acerca de educación superior, con énfasis en temas evaluación, acreditación y globalización. e-mail: jose.sobrinho@prof.uniso.br

Resumen: El texto sustenta que la globalización económica, capitalista e informacional impone importantes transformaciones a la educación superior, notablemente los cambios relativos a las misiones institucionales y los desplazamientos de las referencias centrales: de las humanidades a las disciplinas técnicas y de la sociedad al mercado. Estos cambios ponen en relieve la centralidad de los temas de calidad en las políticas y prácticas educativas. La ideología de la acumulación neoliberal impone a la educación superior la función de motorizar la economía por medio de producción y transmisión de conocimientos "útiles", instrumentales, en general de base técnico-informática. Esta corriente ideológica crecientemente más hegemónica en los sistemas internacionales de evaluación y acreditación relaciona la calidad al cumplimiento de los papeles estratégicos que a la educación superior correspondería desempeñar en la actual fase de la economía global. En contra a esa corriente y sustentando la primacía de la ética, de la pertinencia y de la relevancia social, el texto plantea que la calidad debe estar referida a las finalidades centrales de la educación superior: a los proyectos y procesos de emancipación de los individuos y de las comunidades humanas en todas dimensiones, a la formación integral de ciudadanos-profesionales portadores de conocimientos y valores fundamentales a la vida social y a la construcción de sociedades justas y desarrolladas económica y culturalmente.

Palabras clave: Educación superior. Calidad. Evaluación. Acreditación. Innovación tecnológica. Pertinencia.

\section{POLÍTICAS E CONCEITOS DE QUALIDADE: DILEMAS E DESAFIOS}

Resumo: O texto sustenta que a globalização econômica, capitalista e informacional impõe importantes transformações à educação superior, notadamente as mudanças relativas às missões institucionais e os deslocamentos das referências centrais: das humanidades às disciplinas técnicas, e da sociedade ao mercado. Estas mudanças põem em relevo a centralidade dos temas da qualidade nas políticas e práticas educativas. A ideologia da acumulação neoliberal impõe à educação superior a função de motorizar a economia por meio de produção e transmissão de conhecimentos úteis, instrumentais, em geral de base técnico-informática. Esta corrente ideológica crescentemente mais hegemônica nos sistemas internacionais de avaliação e acreditação relaciona a qualidade ao cumprimento dos papéis estratégicos que à educação superior corresponderia desempenhar na atual fase da economia global. Contra essa corrente e sustentando a primazia da ética, da pertinência e da relevância social, o texto sustenta que a qualidade deve estar referida às finalidades centrais da educação superior: aos projetos e processos de emancipação dos indivíduos e das comunidades humanas em todas as dimensões, à formação integral de cidadãos-profissionais portadores de conhecimentos e valores fundamentais para a vida social e a construção de sociedades justas e desenvolvidas econômica e culturalmente.

Palavras chave: Educação superior. Qualidade. Avaliação. Inovação tecnológica. Pertinência.

\section{POLICIES AND QUALITY CONCEPTS: DILEMMAS AND CHALLENGES}

Abstract: This paper argues that economic globalization, capitalist and informational imposes significant changes to higher education, especially changes concerning institutional missions and displacements 


\begin{abstract}
of central references: from the humanities to technical disciplines, and from society to market. These changes bring out the centrality of issues of quality in educational policy and practice. The ideology of neoliberal accumulation imposes on higher education the function of powering the economy through the production and transmission of useful and instrumental knowledge, generally of technical or computer based nature. This ideological trend, increasingly more hegemonic in international evaluation and accreditation systems, relates quality to compliance with the fulfillment of the strategic roles of higher education in the current phase of the global economy. Against this current and upholding the primacy of ethics, pertinence and social relevance, the paper argues that quality should be referred to the central purposes of higher education: the projects and processes of emancipation of individuals and human communities in all dimensions, the integral education of citizens and professionals with knowledge and values that are fundamental to social life and to the building of societies that are economically and culturally developed.
\end{abstract}

Keywords: Higher education. Quality. Evaluation. Technological innovation. Relevance.

\title{
INTRODUCCIÓN
}

Para mejor comprender los conceptos y las políticas de calidad vigentes en el campo de la educación superior, es necesario tener en cuenta el escenario de la globalización económica y sus principales impactos sobre la política y el mundo cultural. En especial, es importante conocer las principales transformaciones que la globalización impone a la educación superior, notablemente los cambios relativos a las misiones institucionales. Las metamorfosis de los fines de la educación, ahora más explícitamente orientados a los intereses económicos, producen grandes cambios en la cultura académica. La búsqueda de la verdad, valor histórico de la ciencia y fundamento importante del desarrollo humano integral, cede el paso a la producción de conocimientos útiles al desarrollo restrictivamente económico. La privatización y el casi-mercado, con sus pesadas consecuencias, se constituyen como las tendencias centrales de la educación superior en estos tiempos de globalización. En ese escenario, adquiere relieve la innovación tecnológica, presuntamente un poderoso motor del progreso. Estos cambios moldean los conceptos de calidad y las políticas de aseguramiento de la calidad en el campo de la educación superior. Por ello, es necesario rescatar los significados sociales de la formación humana y la coesencialidad que hay entre calidad y pertinencia.

\section{ESCENARIO CAMBIANTE DE LA EDUCACIÓN SUPERIOR}

La actual globalización que vivimos extensiva e intensivamente desde las últimas décadas del siglo XX es un proceso histórico de múltiples dimensiones que mezcla en interacciones mutuas y relaciones de interdependencias la eco- 
nomía, la política, las convivencias personales, los sistemas de comunicación, la ciencia, la cultura, la educación, desde los micro-fenómenos de la vida privada hasta los fenómenos transnacionales y supranacionales. La marca esencial de nuestro tiempo es la globalización económica. La nueva orden económica ha ido produciendo notables transformaciones en los distintos sectores de la vida humana alrededor del mundo, como, por ejemplo, una nueva división internacional del trabajo, interdependencia de los mercados, utilización intensiva y extensiva de las tecnologías de información, liberalización y flexibilización en las organizaciones empresariales, cambios en las relaciones entre capital y trabajo, desempleo estructural, aumento de las desigualdades sociales y de las asimetrías entre países, disminución del Estado en la provisión de la educación pública y de la justicia social y consecuente expansión del mercado y de la oferta transnacional de educación superior (DIAS SOBRINHO, 2005, p. 22-23).

Los procesos de globalización están impulsando importantes transformaciones en todas las dimensiones de la vida humana. Economía, política, relaciones interpersonales, Estado, mundo laboral, valores, comercio, nuevas formas de producción del conocimiento, estructuras de información y comunicación, todo está cambiando rápidamente y generando contextos de alta complejidad. (DIAS SOBRINHO; DIAS, 2009, p. 327).

El contexto global lleno de contradicciones, de incertidumbres y rápidos cambios tiene importantes impactos en la educación superior. Los notables avances en los campos de ciencia y tecnología, particularmente en la tecnología de información, expanden considerablemente las condiciones de producción de conocimientos y de aprendizaje. Al mismo tiempo, los cambios en el modo de producción y la expansión de los medios informáticos engendran una fantástica explosión epistémica cuyo dominio completo en el campo académico se vuelve imposible. Hoy en día la cantidad de conocimientos disciplinares se duplica en algo como dos meses o poco más, lo que también indica estar ocurriendo una vertiginosa obsolescencia de los saberes y de las técnicas.

Las transformaciones en la educación superior no son solamente de naturaleza cuantitativa y estructural. Son también cambios cualitativos. $\mathrm{La}$ cuestión central respeta a los fines: la educación superior está dejando de tener como referencia el desarrollo integral de la sociedad y la formación de la conciencia crítica de ciudadanos y crecientemente va asumiendo los papeles de instrumentación técnica, competencia individual e instrumentalización económica. Cambian los sentidos de los conocimientos y de la formación. Ahora tienen valor sobre todo los conocimientos útiles a la economía de 
mercado; a su vez la formación humana se reduce casi exclusivamente a la capacitación laboral.

Visiones de mundo conflictivas constituyen maneras distintas de pensar y hacer la educación superior. A las instituciones educativas se les impone la exigencia de dar respuestas rápidas y satisfactorias a problemas y demandas de amplias implicaciones y múltiples contradicciones. Por ejemplo: producción de alta tecnología, capacitación en áreas profesionales de tipo nuevo, formación para la innovación, impulso a las empresas transnacionales, inserción en el mundo globalizado, educación a lo largo de la vida, preservación de la alta cultura y recuperación de la cultura popular, formación de profesores y de investigadores, promoción de la ciudadanía y fortalecimiento de la democracia, apoyo a la agricultura y a pequeños productores, tecnología de bajo costo, atención a la salud, etc. (DIAS SOBRINHO, 2002, p. 14).

De un lado, como principio moral y político, la educación superior es entendida como un bien público y derecho humano fundamental. Su referencia central sería, pues, la sociedad, el bien común, el desarrollo integral y sustentable de las comunidades humanas según principios de equidad y justicia social. La sociedad del conocimiento llevaría a suponer que un mayor número posible de personas de todas las partes del mundo pudiesen apropiarse de los conocimientos con espíritu público y social. En este caso, el conocimiento sería comprendido como un bien social y un patrimonio de la humanidad que podría dar un sentido más humano y más amplio al desarrollo civilizador.

En contraposición, una corriente hoy hegemónica en muchos países defiende y practica la educación superior como negocio y servicio en beneficio de los intereses privados. Los conocimientos "útiles", instrumentales, en general de base técnico-informática, son los principales motores de la economía. La llamada sociedad del conocimiento es determinada por la sociedad económica global, que impone una disciplina común en base a la información.

La reestructuración de la economía capitalista global es impulsada fuertemente por las tecnologías de información. Conocimiento de base tecnológica y economía neoliberal tienen en tal contexto una estrecha relación. La revolución tecnológica disparada en los años 1970 ejerce un fuerte impacto sobre la economía, la producción y la apropiación de los conocimientos y de las técnicas y las veloces, amplias y profundas transformaciones que se procesan en todas las dimensiones de la vida humana.

La sobrevaloración de los aspectos mercantiles tiene como contrapunto un empobrecimiento de los valores públicos y sociales, al mismo tiempo que fortalece la ideología del individualismo, del darwinismo social, del lucro, 
del consumismo. El modelo prominente de educación superior resulta de transformaciones importantes en su relación con el Estado y con la economía. Instrumentalización económica, entrenamiento y capacitación profesional según determinaciones del mercado, diversificación de fuentes presupuestarias y de proveedores nacionales y transnacionales, casi-mercado, vinculación con sectores productivos, flexibilización curricular, apropiación mercantil de los conocimientos, expansión de empresas educativas y aumento de mecanismos de control y regulación son algunos aspectos constitutivos de ese modelo.

Un cambio de gran significado para la educación superior consiste en una creciente pérdida de valor de los objetos físicos ante el capital intelectual. El capital humano, inmaterial, deviene un activo financiero con alto valor de mercado. Por ello, la educación superior adquiere enorme importancia en el contexto de la sociedad del conocimiento y de la información, debido al hecho de que ella contribuye fuertemente para la globalización de la producción, aumento de la productividad y de la competitividad, y por esto interfiere decisivamente en los destinos de las empresas meta-nacionales y de las sociedades en general. Esto también explica la importancia que las universidades más en línea con las tendencias actuales de la globalización económica atribuyen a la innovación tecnológica. Las instituciones modernas tienen que invertir en el nuevo más allá del nuevo, en un contexto de crecientes novedades e incertidumbres.

La globalización económica neoliberal ha generado un desplazamiento de las referencias centrales de la educación superior: de la sociedad al mercado y de las humanidades a las disciplinas técnicas. Esta economía hace prevalecer un universalismo de base técnica, cuyas disciplinas son consideradas "ricas", pues presentan elevadas ventajas competitivas en las disputas por financiamiento y prestigio social y están vinculadas a los intereses empresariales (DIAS SOBRINHO, 2010, p. 1234).

La educación superior no logra responder satisfactoriamente a todas las demandas complejas y frecuentemente contradictorias en esta fase de la globalización. La universidad no está sabiendo explicar a sí misma, a la sociedad y al Estado qué tipo de institución debe ser y es capaz de ser, para responder eficaz y coherentemente a las demandas cambiantes y desconectadas que le son impuestas por distintos sectores locales y mundiales en un cuadro de crisis de valores y de incertidumbres con relación al futuro. La fantástica explosión epistémica, la rápida obsolescencia de conocimientos y asimismo las exigencias más y más complejas de capacitación intelectual y profesional superan en mucho la capacidad de respuesta de las instituciones educativas. Además, países pobres o en desarrollo deben solventar o al menos amenizar los déficits educativos y 
las desigualdades sociales y, al mismo tiempo, enfrentar los imperativos de la competitividad del mundo globalizado.

Según la ideología ampliamente diseminada y en algún sentido confirmada en la realidad competitiva del mundo globalizado, sólo pueden competir a nivel internacional los países que tienen poder tecnológico. La inserción creativa en la sociedad del conocimiento exige adecuadas estructuras físicas y vigorosas inversiones en la formación tecnológica, pero los países pobres tienen inmensas dificultades para solventar los históricos atrasos en ese sector. Un tipo de división internacional con alto poder de determinación sobre el desarrollo económico y social consiste en la dualidad entre los inforricos y los infopobres, o sea, entre los que tienen un alto desempeño en las nuevas tecnologías de información y los analfabetos digitales. Conocimiento e información son capitales preciosos del desarrollo y de inclusión social. Pero, es de lastimar que en muchos países la mayoría de la populación está desprovista de los medios de adquisición de conocimientos y de información. Esto significa que la sociedad del conocimiento y de la información no está siendo una sociedad para todos.

\section{INNOVACIÓN TECNOLÓGICA Y CALIDAD}

La innovación tecnológica tiene un rol fundamental para el desarrollo humano justo y sustentable. Entre tanto, como también se puede afirmar de la propia ciencia, no toda innovación porta valores favorables a la humanización y al desarrollo civilizador. En este apartado se contraponen dos conceptos alternativos de innovación: por un lado, una noción y su respectiva práctica de innovación basadas en el cientificismo y en la ideología neoliberal; por otro lado, la idea de que la innovación tecnológica ha de estar necesariamente vinculada a la pertinencia y a la relevancia sociales.

La ideología de la acumulación neoliberal instituye la innovación tecnológica como una dimensión estratégica de los nuevos papeles que la universidad debe desempeñar en la actual fase de la economía global. La innovación determinada en el contexto capitalista de incesante búsqueda de lucros es considerada una importante palanca del progreso. La universidad es elegida ahora el locus privilegiado de la innovación tecnológica y ésta se constituye en instrumento central del desarrollo económico y de la competitividad. Ya son muy comunes en las universidades algunas oficinas de gestión de proyectos, transferencia de tecnologías y vinculación con instancias gubernamentales y con los sectores productivos y de servicios. Estas instancias administrativas son muy importantes 
para organizar y dinamizar los proyectos de innovación tecnológica y promover las articulaciones con los medios empresariales.

Es muy preocupante la tendencia predominante en muchas universidades públicas a atribuir a la innovación un carácter destacadamente técnico-económico en atención casi exclusiva a los imperativos empresariales. Diferentemente de las actuales tendencias, el referente principal de la innovación tecnológica desarrollada en las universidades públicas debería ser la sociedad toda, o sea, el desarrollo humano integral, y no restrictivamente el mercado.

Además, la vinculación estrecha de las innovaciones tecnológicas con el sector industrial refuerzan la primacía asignada a las áreas que más producen conocimientos, patentes y profesionales de alto valor económico en detrimento de las humanidades, donde en general están las disciplinas más reflexivas y críticas. Ocurre hoy un desplazamiento de la referencia central de la universidad, con implicaciones en las jerarquías del conocimiento y consecuentemente en las estructuras del poder académico-universitario. El universalismo de base técnica y motorizado por la innovación tecnológica ahora prevalece sobre las áreas de humanidades. En buena parte definidas en círculos académicos de gran reconocimiento y legitimadas por actores nacionales, las disciplinas técnicas guardan estrechas alianzas con las grandes empresas y laboratorios transnacionales y, por ello, son las que tienen más valor y prestigio, son más competitivas y las que reciben más elevados fondos públicos y privados.

La labor académica adquiere crecientemente un sentido de mercado y se transforma en motor del desarrollo económico basado en el "paradigma tecnoinformático-educativo" (DIDRIKSSON TAKANAYAGUI, 2000, p. 12). El docente universitario se ve obligado a asumir funciones que normalmente son propias de sectores mercantiles y el histórico ethos académico paulatinamente pierde su valor autonómico y se subsume en las relaciones de fuerza del mercado.

Teniendo en cuenta las exigencias de confidencialidad, los derechos de propiedad, la necesidad de adecuación a las empresas, muchas veces las innovaciones tecnológicas escapan de la autoridad de los colegiados académicos y no tienen en cuenta las posibles consecuencias prácticas y morales que podrían generar en el campo social. Esta visión instrumental de las ciencias duras, que cree en la neutralidad del conocimiento objetivo y en el papel del complejo ciencia-tecnología como motor del progreso irreversible y siempre creciente de la humanidad, es basada en algunos mitos de inspiración positivista-cientificista, así nombrados por Sarewitz (1996 apud DIAS SOBRINHO; DIAS, 2009, p. 335): 
1. Mito del beneficio infinito: cuanta más ciencia y tecnología, más progreso y bienestar social;

2. Mito de la investigación libre: cualquier línea de investigación orientada a la comprensión de los procesos fundamentales de la naturaleza rendirá beneficios para la sociedad, como cualquier otra investigación científica;

3. Mito de la responsabilidad: los mecanismos de control de la calidad de la investigación científica (revisión por pares, fidelidad al método científico, por ejemplo) asegurarían las principales responsabilidades éticas del sistema de investigación;

4. Mito de la autoridad: la información científica ofrecería una base estrictamente objetiva para la resolución de disputas políticas;

5. Mito de la autonomía: el conocimiento generado en la "frontera" de la ciencia sería autónomo con relación a sus consecuencias prácticas y morales en el ámbito social.

Es indudable que las empresas son actores de enorme importancia en la sociedad capitalista. Por ello, es razonable que la universidad establezca relaciones de cooperación con ellas. Pero no es aceptable que la universidad sea sólo un instrumento de acumulación capitalista en favor de pocos y en detrimento de la mayoría de la sociedad. Tampoco es creíble que la ciencia y la tecnología puedan triunfar sobre todas las carencias sociales e impulsar un imparable y siempre positivo progreso de la sociedad humana.

En lugar de privilegiar los intereses privados de empresas y de individuos, la innovación tecnológica en las universidades públicas debería estar integrada a las estrategias de desarrollo humano y social sustentable. Esto significa que la innovación debería formar parte de los procesos formativos, contribuir al avance de la ciencia y de la tecnología y ser pertinente a las comunidades. La relación universidad-empresa habría de ser apenas un aspecto de una relación más amplia de la relación universidad-sociedad. La esencia de la universidad es formativa con relación a los individuos y a la sociedad. Por lo tanto, la innovación universitaria no puede eludir su valor social y educativo, para allá de su función operacional ceñida a las empresas.Además de ello, no se puede olvidar que el modelo de ciencia y tecnología que se adopta en países periféricos imita, en muchos casos, la agenda de investigación de los países centrales, como si hubiera una ciencia neutra y universal, incontaminada ideológicamente e independiente de los contextos, que tuviese la potestad de transformar indiferentemente a toda y cualquier sociedad. No ha de ser la ganancia económica el 
valor central de la humanidad, sobre todo si ella beneficia a pocos en detrimento de la mayoría de la populación.

La innovación en la universidad debería privilegiar las tecnologías sociales y poner en práctica el concepto de "aprendizaje social". Este modelo crea la integración entre distintos actores internos y externos, rompiendo con las prácticas ofertistas de enseñanza y construcción de conocimientos limitados a una sola disciplina. En ello, profesores y estudiantes de diferentes áreas y ciudadanos de distintos segmentos de la sociedad se vinculan en la construcción de espacios sociales de aprendizaje, producción e intercambios de conocimiento.

La innovación con sentido social y público puede de esta manera integrar interdisciplinarmente las tres estructuras universitarias: enseñanza, investigación y vinculación con la sociedad. Ella produce una ecología del saber al crear estructuras de cooperación y articular los esfuerzos de los actores de la universidad y de la sociedad en general. El saber socialmente producido se enriquece en la medida que esté vinculado a las necesidades sociales y más democráticamente sea apropiado por los ciudadanos de una comunidad.

La vinculación entre enseñanza e investigación y entre la universidad y la sociedad debe tener un carácter democrático y de permanencia, no resumiéndose a acciones esporádicas y pasajeras. Estos cambios exigen acciones y actitudes innovadoras en la docencia y en la formación continua de los docentes-investigadores. Además del dominio de su área, el docente debe buscar construir, en los procesos de innovación vinculados al entorno de la universidad, una interacción colaborativa con los estudiantes y miembros de la comunidad externa, de forma tal que su labor sea beneficiosa a la creación de espacios de reflexión y comunicación con alta potencialidad de formación personal y desarrollo social.

No olvidar que enseñanza no es sólo instrucción y que la información es sólo una parte de la formación. Más importante que la enseñanza es lo que se aprende. Más fundamentales y duraderos que los contenidos disciplinarios son los valores. Son los valores los que forman. "Son los valores los que transforman la simple instrucción en educación y el conocimiento en sabiduría" (TÜNNERMANN, 2009, p. 269).

La innovación en la universidad no debería alejarse del sentido público de la educación. No debería, pues, tener como foco principal la competitividad económica y comercial. El compromiso con la educación pública exige identificar prioridades de enseñanza e investigación no sólo inclinándose al desarrollo de las empresas y a la apropiación privada, sino sobre todo orientadas al bienestar de las comunidades sociales, especialmente de los sectores marginados. 
Existen en las sociedades de países periféricos renitentes problemas y rezagos para cuya solución, o al menos para su minoración, las actividades de innovación con sentido público pueden contribuir. Los problemas más acuciantes de las sociedades que todavía no alcanzaron altos niveles de desarrollo humano están interconectados: pobreza, hambre, analfabetismo, violencia, desempleo, exclusión, deterioración ambiental etc.

\section{DISTINTAS CARAS DE LA CALIDAD DE LA EDUCACIÓN SUPERIOR EN DISPUTA}

Las consideraciones anteriores forman la moldura para una reflexión atenta sobre los significados más corrientes de la calidad de la educación superior. La primacía del económico ha producido un importante cambio semántico en el concepto de calidad de la educación superior. En base a una racionalidad y a una metodología apropiada de la industria, la calidad está predominantemente asociada a indicadores de desarrollo económico, tasas de crecimiento, números de productos, rendimientos estudiantiles, capacidad de captación de recursos, sustentabilidad financiera etc.

La calidad está, de esta manera, identificada a los resultados de una aplicación de los indicadores establecidos por aseguradores de la calidad. Según esa racionalidad, una educación de calidad es aquella que propicia a los individuos y a las empresas las capacidades competitivas adecuadas y necesarias a los cambios producidos por la globalización de la economía capitalista y su ideología. Cuanto más sirve al desarrollo de las empresas y al fortalecimiento de la ideología del éxito individual, más calidad tendría una educación superior, en esa racionalidad neoliberal.

Los gobernantes, en general, acordes con la ideología del capitalismo global, y coherentes con la idea de que la educación superior deba ser un instrumento del progreso del país mediante el desarrollo de las empresas, operan en el sentido de construir un sistema eficiente y regulado por mecanismos de evaluación y acreditación. Por ello, crean organismos de evaluación y control de la calidad y un amplio aparato de instrumentos legales y normativos, flexibilizando la autonomía de los medios y aumentando el control sobre los resultados. Para los operadores gubernamentales, la calidad de la educación superior se mide sobre todo por desempeños de estudiantes en exámenes nacionales y asimismo por la ampliación de la matrícula, disminución de abandonos, desarrollo de competencias y habilidades laborales, capacidad de obtención de empleos, eficiencia y eficacia administrativa. 
Es más fácil y cómodo lograr acuerdos respecto a aspectos técnicos, metodológicos y dimensiones objetivas de la calidad. Expertos en acreditación, muchos de ellos pertenecientes a organismos multilaterales, legitimados por la comunidad académica internacional y por gestores nacionales, establecen objetivamente los conceptos y estándares de calidad. En esta perspectiva, calidad corresponde al cumplimiento, en distintos niveles, de los estándares acordados por expertos (autoridad intelectual) y legitimados por las agencias de evaluación y acreditación (autoridad política). Además, esa modelación objetiva de la calidad ofrece informaciones interesantes a posibles clientes (estudiantes y familiares) y al Estado. En esta cuestión se involucran intereses de los demandantes de empleos y de los empleadores de las esferas privada y pública. De las demandas de los clientes (mercado) y de las obligaciones de autoridad pública (Estado), con relación a rendición de cuentas e información a la sociedad, resulta que la evaluación de la calidad progresivamente se transforma mucho más en una herramienta de control que una herramienta de mejora (valor prioritario de los académicos).

Todos estos indicadores predominantemente objetivos son muy importantes al Estado y fácilmente comprendidos por los formadores de opinión y por eventuales "consumidores" de la educación superior, sobre todo cuando son representados en índices, estadísticas y rankings. Ellos sirven muy bien al modelo hegemónico que entiende la educación como un servicio a la competitividad económica y obligan a los profesores y administradores a asumir aptitudes y practicar mecanismos que son más adecuados al mundo competitivo del mercado, como si fueran trabajadores de empresas comerciales. La racionalidad empresarial hace creer que la gestión de desempeños per se producirá inevitablemente la calidad, es decir, la satisfacción de los clientes. Si el mercado se entiende como canjes de servicios y bienes basados en el precio, la educación se transforma en servicio y mercadería para beneficio privado.

El creciente fetichismo de la eficiencia y de la eficacia lleva a entender la calidad de la educación por su relación con el mercado. De ahí el uso de temas e instrumentos analíticos propios de medidas de calidad en el sector del mercado: relación entre formación profesional y empleo, crecimiento de matrículas, proporción profesor/alumno/funcionario, costes en relación al PBI, presupuestos, financiación, inputs, outputs, tiempos de conclusión, planificación, rentabilidad, productividad etc. La calidad sigue la lógica de la eficiencia y de la eficacia: reducir costes y producir más. En esa perspectiva economicista, una institución educativa tendría más calidad en la medida que logra presentar buenos desempeños en indicadores con respecto a la cantidad de titulaciones, 
número de profesores doctores y de tiempo completo, inversiones, rendimientos estudiantiles, capacidad de captación de recursos financieros, tiempos medios de estudios, tasas de acceso, tomas de decisión, contexto demográfico, económico y social, resultados de la enseñanza con relación al mercado de trabajo etc. La evaluación en esa lógica es sobre todo un instrumento de medición de la responsabilidad contable, de eficacia y de competencia en el mercado.

Sin grande preocupación con los beneficios sociales y públicos, con respecto a la formación, la calidad educativa sometida a la ideología del mercado tiende a ser aquella que más desarrolla las competencias individuales y empresariales. De ahí la importancia del uso de indicadores de desempeño de aprendizaje, medido por medio de exámenes nacionales, y desempeño organizacional, indicado por instrumentos de medida de eficiencia (proporción alumnos/profesores/ funcionarios, costes etc) y de eficacia (números de graduados y de empleos, por ejemplo). En los dos casos, el foco principal está en los resultados.

Para allá de desempeños en exámenes y además de contenidos disciplinares que rápidamente caducan, para allá del valor social objetivo (empleo, desarrollo de la sociedad, acumulación de conocimientos útiles a la economía etc), la educación tiene relación con aprendizajes de valores, autoestima, aptitudes, proyectos y expectativas personales y colectivas (valor social subjetivo). Como los distintos actores internos y externos de la universidad tienen diferentes y muchas veces contradictorios valores y objetivos, los conceptos de calidad no son unánimes. Tampoco la calidad podrá ser identificada y quizás mensurada solamente por algunos de sus elementos más visibles.

Aunque la calidad no se confunda con las condiciones objetivas de producción, difícilmente ella se realizará sin adecuados medios, o sea, sin organización apropiada, buenos laboratorios, bibliotecas, docentes calificados, gestión eficaz etc. La calidad en educación es un fenómeno complejo que relaciona dimensiones pedagógicas, científicas, técnicas, políticas, infraestructurales, culturales, económicas, éticas, morales etc.

La calidad en educación no se ciñe a elementos técnicos y objetivos, como, por ejemplo, una buena infraestructura y cuerpo profesoral con elevada titulación, aunque esto también sea mucho importante. Enseñanza, personal, equipamientos, salud financiera, administración eficiente son condiciones de producción de la calidad. Importante subrayar que estos son medios, sin duda, imprescindibles, pero la calidad no se resume a esto. La calidad debe estar referida a las finalidades centrales de la educación superior, o sea, a la formación integral de ciudadanos-profesionales portadores de conocimientos y valores fundamentales a la vida social y a la construcción de una sociedad justa 
y desarrollada económica y culturalmente. En otras palabras, educación de calidad es aquella que cumple satisfactoriamente su función de contribución a los proyectos y procesos de emancipación de los individuos y de las sociedades.

Es bien cierto que se puede atribuir valores comunes y universales a la educación superior: búsqueda de la verdad, rigor científico, libertad de pensamiento, respeto a la diversidad, democracia, justicia social etc. Estos valores deben hacer parte de la misión de toda institución educativa y, por lo tanto, son esenciales al concepto de calidad. Pero, además de valores universales, las instituciones educativas han de tener en cuenta las realidades y las culturas nacionales, las demandas y necesidades de las sociedades en donde están insertadas. En otras palabras, deben ser pertinentes. Sin pertinencia, o sea, sin un compromiso con la sociedad y en especial con sus demandas y necesidades de formación y de desarrollo humano integral, una institución educativa no cumple plenamente las exigencias de calidad.

Formación humana integral se refiere a procesos de desarrollo de la autonomía de los sujetos en todas sus dimensiones indisociables: económicas, epistemológicas, intelectuales, morales, éticas, estéticas, afectivas, sociales, profesionales. Decir que la calidad tiene la pertinencia social como uno de sus elementos esenciales implica en entender que la calidad es un concepto relativo, dinámico y siempre referente a realidades concretas y a proyectos que los individuos y las sociedades construyen en sus procesos de vida. En este sentido, una institución educativa de calidad tiene que presentar relevancia científica y pertinencia social en sus procesos de conocimiento y formación. La relevancia científica tiene a ver con la importancia y el rigor de los conocimientos con relación al avance de la ciencia y con la formación de individuos y sociedades cultural e intelectualmente ricos. Pero la ciencia solo tiene sentido cuando contribuye para la formación humana integral y, por lo tanto, para los procesos de construcción jamás acabada del bien humano.

Un concepto academicista, o sea, incontaminado, universal, estático y abstracto de calidad no logra dar cuenta de todas las especificidades y realidades idiosincrásicas de las instituciones de educación superior y de sus compromisos y potencialidades con relación a los conocimientos, a los requisitos internacionales, a las demandas de la sociedad nacional y de la comunidad local. Lo que se entiende por calidad y lo que se plantea como estándares de calidad en los países ricos no necesariamente se corresponden en plenitud y sin adaptaciones a los países en desarrollo y a sus universidades. Las situaciones históricas, sociales, culturales y asimismo los retos y desafíos de las principales potencias técnicocientíficas centrales y los países periféricos son distintos y, por consiguiente, 
distintos deben ser los compromisos y las estrategias de las respectivas instituciones. Entonces, la evaluación de la calidad no debería seguir exactamente los mismos criterios y los mismos indicadores sin cualquier preocupación en insertarlos en los contextos sociales concretos.

Los significados de pertinencia son diferentes para sociedades industrialmente desarrolladas y para las populaciones de países pobres o emergentes. Los países ricos demandan ciencia y tecnología para motorizar el desarrollo de las industrias y grandes corporaciones transnacionales. Para ellos, su modelo societario es el modelo que debe ser universalmente reconocido. Desde ese punto de vista, calidad es sinónimo de desarrollo y resulta de la adecuación a los estándares de las grandes potencias. Estos estándares, conceptos y criterios de la big science son subsidiarios de las grandes corporaciones económicas e impuestos a las comunidades académicas e instancias gubernamentales de países periféricos como si fuesen modelos universales y tuviesen la potestad de impulsar imparablemente un progreso siempre beneficioso para la humanidad.

No todos los criterios de los centros hegemónicos son adecuados a las naciones periféricas. Éstas, además de los requisitos del desarrollo económico, también requieren de la educación y de la ciencia una fuerte contribución para la disminución de las desigualdades sociales, para el combate a la miseria, para el aumento de la inclusión y el fortalecimiento de la ciudadanía y de la democracia. El concepto neoliberal de pertinencia, diseminado desde las potencias centrales como una creencia necesaria a toda la humanidad, no sirve a realidades de países que tienen otros problemas apremiantes a solucionar, desde la miseria hasta los derechos humanos y la plena democratización.

Ahora bien, la pertinencia debe tener una fuerte relación con la justicia social, no con los intereses exclusivos del mercado. La capacitación técnica para el trabajo, el aumento de la competencia laboral, el avance de la innovación tecnológica y del conocimiento útil al desarrollo de las empresas y otras funciones relacionadas con la economía son valores sociales objetivos también constitutivos de las misiones esenciales de la educación de calidad. Ellos expresan una faz de la pertinencia y de la relevancia social. No obstante, estos valores sociales objetivos no pueden prevalecer sobre los valores de la equidad que fundamentan las políticas públicas de justicia social.

Mucho más que atención al desarrollo de las empresas, la pertinencia en educación debe portar un valor público y social. Ella se refiere al papel que la educación ha de cumplir relativamente a la sociedad y lo que ésta espera de aquélla (TÜNNERMANN, 2009, p. 268). El desarrollo económico sin justicia social contraría los valores de la democracia, pues produce más exclusión y 
desigualdades sociales. Una sociedad que no se construye sobre el principio moral y político de la equidad es una sociedad dividida, intolerante, inescrupulosa, atravesada de conflictos, violencias de diversos tipos, inseguridades, marginaciones. La injusticia social rompe los tejidos sociales y corrompe las condiciones básicas de una vida digna.

La educación superior, por medio de los conocimientos científicos, pertinentes y socialmente relevantes, al desempeñar sus funciones con sentido público, contribuye para potenciar las sinergias que impulsan el desarrollo sostenible de sociedades. Diferentemente de las empresas, donde la ganancia y la competencia son fines en sí mismos, la naturaleza pública de la educación significa negación de la rivalidad y de la exclusión. Por principio, la educación es un bien público, derecho social, y por consiguiente debe estar disponible a todos. El acceso de una persona a la educación y al conocimiento no debe implicar la exclusión y tampoco la privación de los otros. Al contrario, significa más inclusión y enriquecimiento de la sociedad. (DIAS SOBRINHO, 2008).

El conocimiento público y pertinente es un factor esencial de la formación humana integral, personal y social. Las universidades son instituciones centrales para el esfuerzo conjunto que deberían realizar el Estado y la sociedad a fin de interrumpir el círculo vicioso de la desigualdad socio-educativa. La educación pública debe ser suministrada de forma equitativa, continuada y permanente, sin ninguna discriminación social, comercial y económica (GINKEL; DIAS, 2006, p. 52).

Un referente importante de la calidad de una institución educativa es su empeño en contribuir para una amplia y adecuada escolarización de la populación. En la educación superior hay una larga tradición elitista. La necesaria democratización de la sociedad requiere de estrategias democratizadoras y sustentables de las instituciones educativas consistentes en ampliar las condiciones de acceso y permanencia de las capas históricamente marginadas.

La inclusión de más personas a la sociedad del conocimiento, desde una perspectiva del bien público, no significa exclusión o privación de otras. Cuantas más personas tienen acceso al conocimiento, más rica y justa es la sociedad. La calidad de la educación superior se realiza plenamente sólo cuando una institución alía en alto grado sus funciones científico-formativas con las realidades concretas de la sociedad en la cual se inserta, o sea, cuando conocimiento y formación se enlazan con los proyectos personales y sociales de construcción de la ciudadanía pública que constituyen las bases de la nación.

Aunque la calidad no se confunda con las condiciones objetivas de producción, difícilmente ella se realizará sin adecuados medios, o sea, sin organización 
apropiada, buenos laboratorios, bibliotecas, docentes cualificados, gestión eficaz, etc.

Si los responsables por la administración central de los Estados tienen la autoridad de la regulación y el deber de garantizar el adecuado funcionamiento de las políticas públicas educacionales, son los académicos los actores más cualificados para atribuir valor a las cuestiones académicas que tienen su fulcro en la formación integral. La educación superior tiene enorme valor público. Por ello, la determinación de lo que sea su calidad no es una sencilla cuestión técnica sólo a cargo de expertos y gestores, tampoco es sólo una cuestión académica.

El tema de la calidad tiene indiscutible centralidad en las políticas y prácticas educacionales. Por ello, es un campo de disputas que tiene que ver con la hegemonía de conocimientos, actitudes y valores que constituyen el potencial humano público y social, desde una perspectiva epistemológica, política y ética. La calidad de la educación superior, en última instancia, tiene como referentes fundamentales los valores de la existencia humana, por tanto, de la sociedad humana para cuya construcción contribuyen las actividades de carácter cognitivo y formativo de una institución educativa.

\section{REFERENCIAS}

DIAS SOBRINHO, José. Universidade e avaliação. Entre a ética e o mercado. Florianópolis, Insular, 2002.

DIAS SOBRINHO, José. Dilemas da educação superior no mundo globalizado. Economia do conhecimento ou sociedade do conhecimento? São Paulo: Casa do Psicólogo, 2005.

DIAS SOBRINHO, José. Cambios y reformas en la educación superior. En: TÜNNERMANN BERHEIM, Carlos (Edt.). La educación superior en América Latina y el Caribe: diez años después de la Conferencia Mundial de 1998. Caracas, Venezuela; Cali, Colombia: Pontificia Universidad Javeriana /UNESCO-IESALC, 2008.

DIAS SOBRINHO, José. Democratização, qualidade e crise da educação superior: faces da exclusão e limites da inclusão. Educação e Sociedade, Campinas, v. 31, n. 113, out/dez. 2010. 
DIAS SOBRINHO, José; DIAS, Rafael de Brito, Innovación en la universidad brasileña. ¿Mecanismo empresarial o estrategia de desarrollo sostenible? En: FERNÁNDEZ LAMARRA, Norberto (Comp.).

Universidad, sociedad e innovación. Una perspectiva internacional. Buenos Aires: EDUNTREF, 2009.

GINKEL, Hans J. A.; DIAS, Marco Antonio Rodrigues. Retos institucionales y políticos de la acreditación en el ámbito internacional. En: LA EDUCACIÓN SUPERIOR EN EL MUNDO EN 2007. Acreditación para la garantía de la calidad y el compromiso social de las Universidades ¿Qué está en juego? Madrid: Mundiprensa, 2006.

DIDRIKSSON TAKANAYAGUI, Axel. La Universidad de la Innovación. Una estrategia de transformación para la construcción de Universidades de futuro. Caracas, Venezuela: UNESCO/IESALC, 2000.

SAREWITZ, D. Frontiers of Illusion: science, technology and politics of progress. Temple University Press. Filadelfia: EEUU, 1996.

TÜNNERMANN, Carlos. Pertinencia y calidad en educación superior. En: FERNÁNDEZ LAMARRA, Norberto (Comp.). Universidad, sociedad e innovación. Una perspectiva internacional. Buenos Aires: EDUNTREF, 2009. 
